\title{
CJK Catalogue:
}

Chinese, Japanese and Korean Catalogue by Belinda Law, Honorary College Librarian CTCA
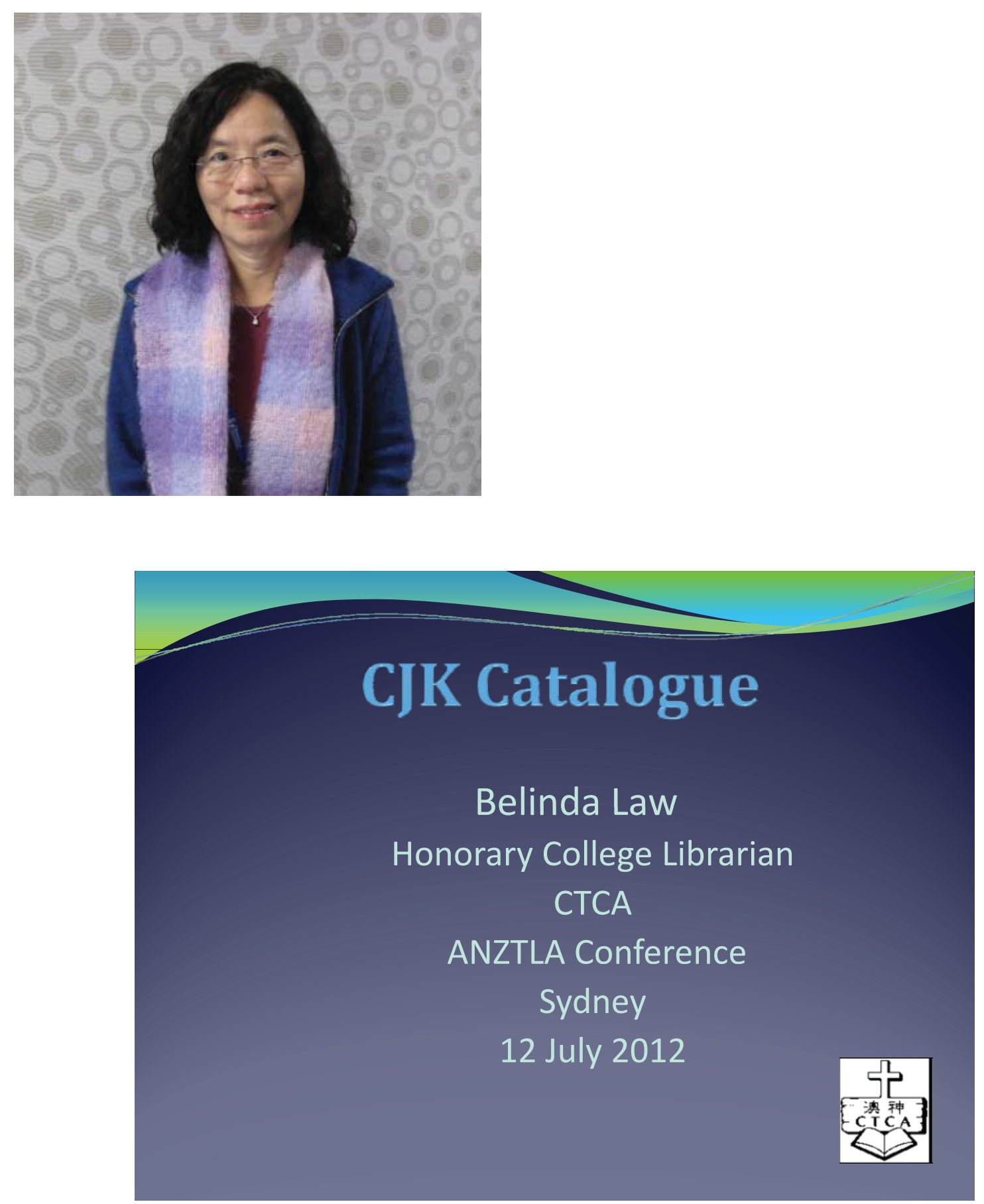


\section{Chinese Theological College Australia (CTCA)}

- Website: http://www.ctca.org.au/

- Location: Office 4C/5 Belmore Street, Burwood 2134 Library Temporary at 2nd floor PTC Library

- History -

- 1991 - Inspired to establish the ministry of the Chinese theological education

- 2000 - Registered as a non-profit religious institute and Certificate programs started

- 2006 - Diploma programs commenced

- 2010 - Registered with ACT, Bachelor of Ministry (BMin) and Master of Divinity (MDiv) commenced

- 2012 - Approval from ACT to teach Graduate Diploma of Theology and Diploma of Ministry [Pathway 2] in Chinese

\section{Objectives}

- to equip Christians to become church pastors that they may serve among the Chinese Churches in Australia

- to equip Christian leaders so as to become the assistance to the pastors

- to provide extended lay theological training to the Chinese Christians in Australia

- to provide in-service further studies for the Chinese pastors in Australia 


\section{Library}

- 2009 - SLS library system installed

- Library set up at West Ryde with approx 200 books

- First commercial order in 2009

- 2010 - moved to Burwood next to PTC

- 2012 - Collection continue to grow, 4000+ books today moved to $2^{\text {nd }}$ floor of PTC Library

- Staff-myself, volunteers and casual staff

\section{SLS integrated library system}

\section{- http://www.sls.org.hk/}

- used by many schools in Hong Kong

- Win Version - WinCirc, WinCAT, WinReport, WinOPAC ImportWizard, WinADMIN, WinINV

for networking

- Web Version - WebOPAC, WebCAT (including importing and reporting function)

for internet 


\section{Computer and system requirement}

- Asian Scripts capabilities - from control panel

- Control Panel - All Control Panel Items ।

Display and input

- System can cope with CJK scripts, such as

- Millennium

- Voyager

- Dynix

- SLS

\section{Record Display in Chinese (OPAC)}

\section{Full Record}

$\square$ Check this checkbox to mark or unmark the current record

\begin{tabular}{|c|c|}
\hline AUTHOR & 鍾志垶。 \\
\hline TITLE & 約翰福音/鍾志垶著. \\
\hline EDITION & 初版. \\
\hline IMPRINT & 香港：天渞毒樓有限公司，2003-2004. \\
\hline DESCRIPTION & 2 开；22 公分. \\
\hline SERIES & 天道聖經註精. \\
\hline NOTE & Includes bibliugraphical references. \\
\hline SUBJECT & Bible. N.T. John -- Commentaries. \\
\hline OTHER TITLE & Colophon title also in English: John. \\
\hline ISBN & 9622085652 (v. 1). \\
\hline ISBN & 9622086039 (v. 2). \\
\hline
\end{tabular}

\section{Location}




\section{Record Display in both Chinese and English (OPAC)}

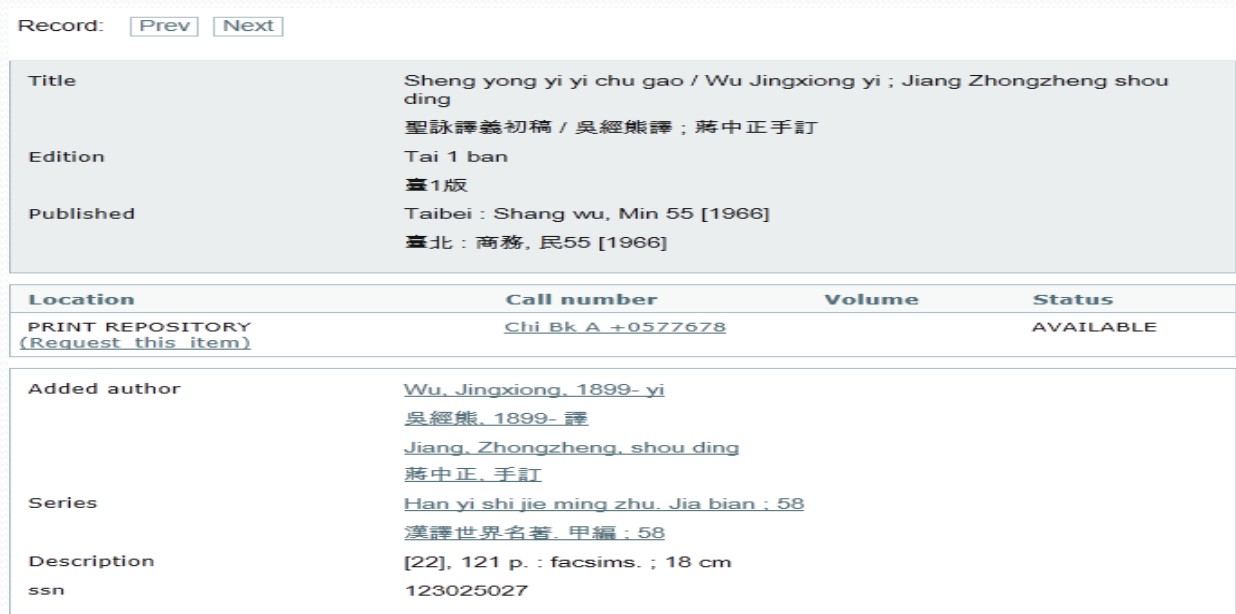

\section{MARC record}

MARC record display

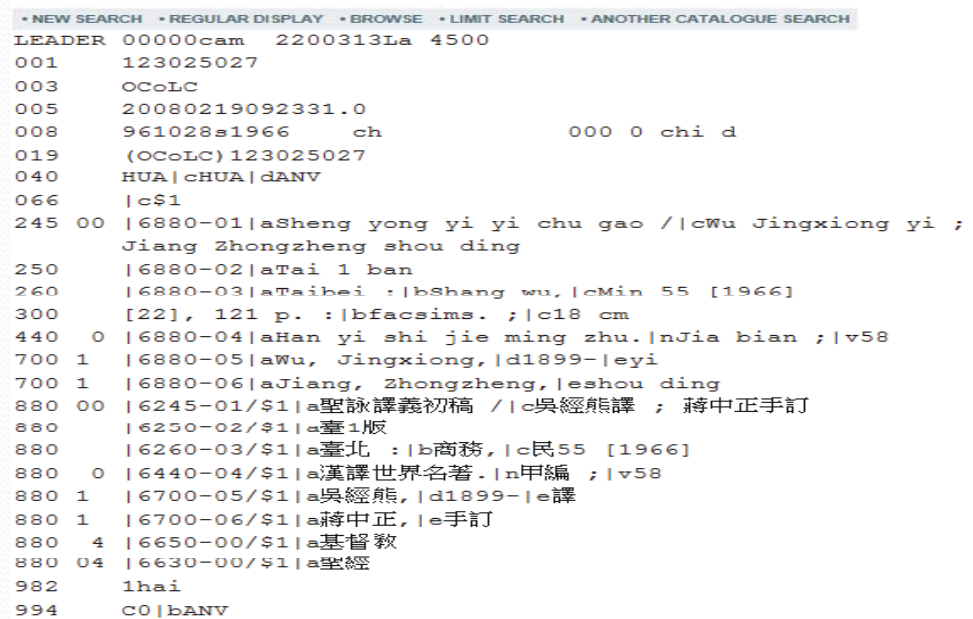




\section{CTCA use SLS}

- OPAC via CTCA Home page

http://sls.ctca.org.au/slsbin/webopac.exe

- Cataloguing module

http://sls.ctca.org.au/slscat/webcat.exe

\section{OPAC}

Chinese Theological College Australia

\section{\begin{tabular}{ll|l|l} 
Advanced Search & Borrowing Record Top 10 Books Report & 中交
\end{tabular}}

\begin{tabular}{|c|c|}
\hline Database Local Catalog & 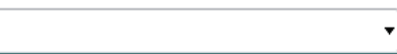 \\
\hline \multicolumn{2}{|l|}{ Search By } \\
\hline Title Exact & Author Exact \\
\hline Title Keyword & Author Keyword \\
\hline Title Stroke & Author Stroke \\
\hline Subject/Discipline & Author Keyword \& Title Keyword \\
\hline $\begin{array}{l}\text { Subject/Discipline \& Reading Level } \\
\text { Call Number }\end{array}$ & $\underline{\text { ISBN/ISSN }}$ \\
\hline
\end{tabular}




\section{Catalogue module}

\section{New Record}

Tools

Logout

中交

Help

Database Local Catalog

\section{Search By}

Title Exact

Title Keyword

Title Stroke

Subject/Discipline

Subject/Discipline \& Reading Level

Call Number

Record Control Number

Advanced Search (Local Catalog Only)

Author Exact

Author Keyword

Author Stroke

Author Keyword \& Title Keyword

$\underline{\text { ISBN/ISSN }}$

Barcode

Accession Number

From Report List File (Local Catalog Only)

\section{Cataloguing Demo}

For downloading records:

- Librariesaustralia http://librariesaustralia.nla.gov.au/

- Login and search from OCLC

- Chinese university library http://library.cuhk.edu.hk/

- For copy and paste

- China Evangelical Seminary - Taiwan

http://wwwlibe.ces.org.tw/webpac2/msearch.dll

- China Graduate School of Theology - EIN

http://www.cgst.edu/en/int8.html 


\section{Select OCLC from Librariesautralia}

rick the databases you wish to search and then click the Save changes button.

\section{Save changes}

These databases can be searched simultaneously:

$\square$ Australian Library Collections (Australian National Bibliographic Database) (more info...)

British Library Catalogue (more info...)

Canada Institute for Scientific and Technical Information (CISTI) (more info...)

Chinese University of Hong Kong (more info...)

Research Libraries UK (RLUK) (more info...)

Informit Collections (more info...)

Hong Kong University of Science and Technology (more info...)

Library of Congress Catalogue (more info...)

National Film And Sound Archive OpenSearch (more info...)

ocl C WorldCat (more info..)

Powerhouse Museum OpenSearch (more info...)

Singapore National Union Catalogue (more info...)

Te Puna (New Zealand National Bibliographic Database) (more info...)

University of Hong Kong (more info...)

National Museum of Australia OpenSearch (more info...)

Search for a book

libraries australia search

Search Advanced Search Browse Terms Search History Saved Records Saved Queries Add Record

\section{Advanced Search}

Find What:

$\underline{\text { Command Search }}$

\begin{tabular}{|l|l|l|l|l|}
\hline Title & starts with & and & Search \\
\hline Subject & Reset \\
\hline Number (eg. ISBN) $\rightarrow$ & all of these & starts with $\square$ \\
\hline
\end{tabular}

Limit to: 


\section{Search Result}

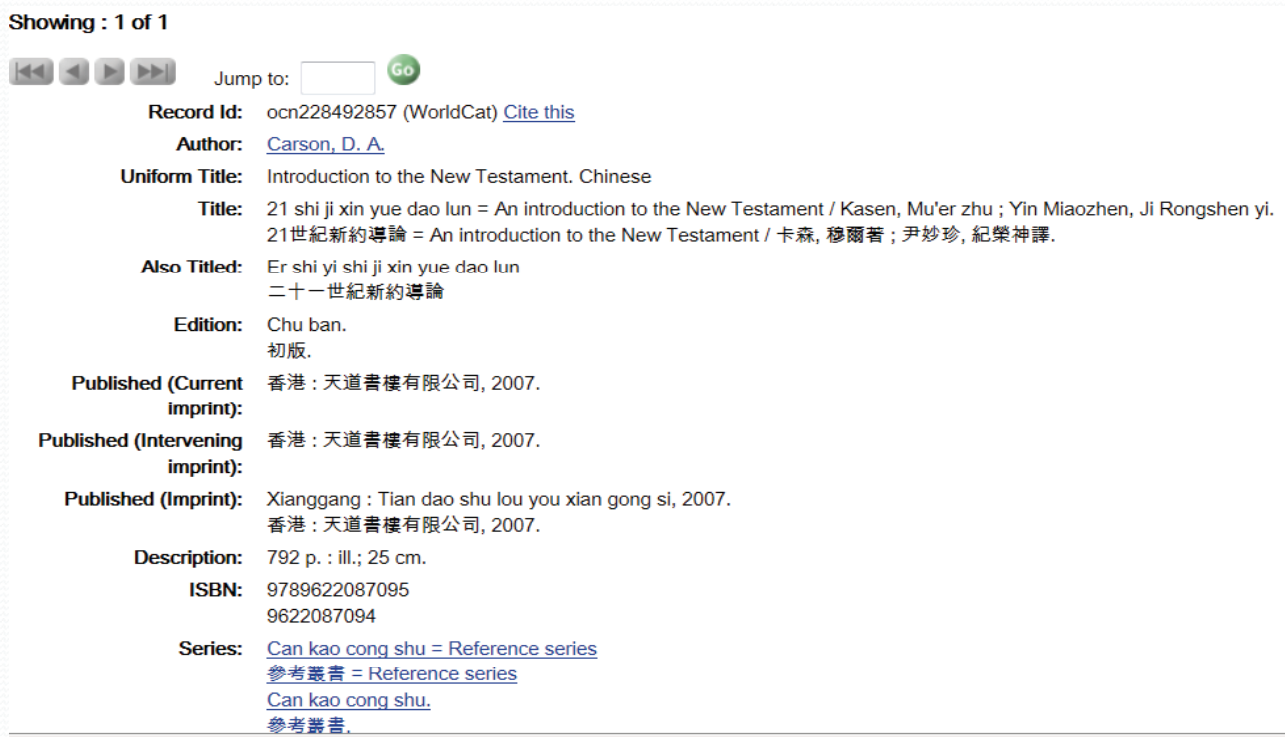

\section{Copy and paste from other catalogue - search}

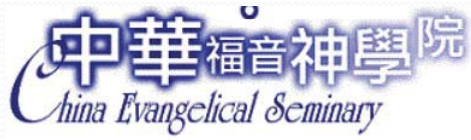

館藏查詢(Library Catalog Search)

關鍵點條件

關鍵字Keyword

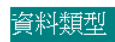

排序方式

書名(Title)

以下檢索條件之查詢結果為符合檢索值開頭的資料

\begin{tabular}{|c|c|}
\hline 書刊名Title & 舊約概觔 \\
\hline 漂題Subject & \\
\hline 登録號Accession Number & \\
\hline 占書ISBN & \\
\hline 刊CODEN & \\
\hline 索書號 & \\
\hline 肖訂欄位\#1(Custom Fields \#1) & \\
\hline 自訂欄位\#3(Custom Fields \#3) & \\
\hline
\end{tabular}

\begin{tabular}{|c|}
\hline 作者Author \\
\hline 出版商Publisher \\
\hline 䠫書名Series \\
\hline 期刊ISSN \\
\hline 西文刊名縮蔫 \\
\hline 分館 / 館藏地 \\
\hline 自訂欄位刑 (Cus \\
\hline
\end{tabular}




\section{Result}

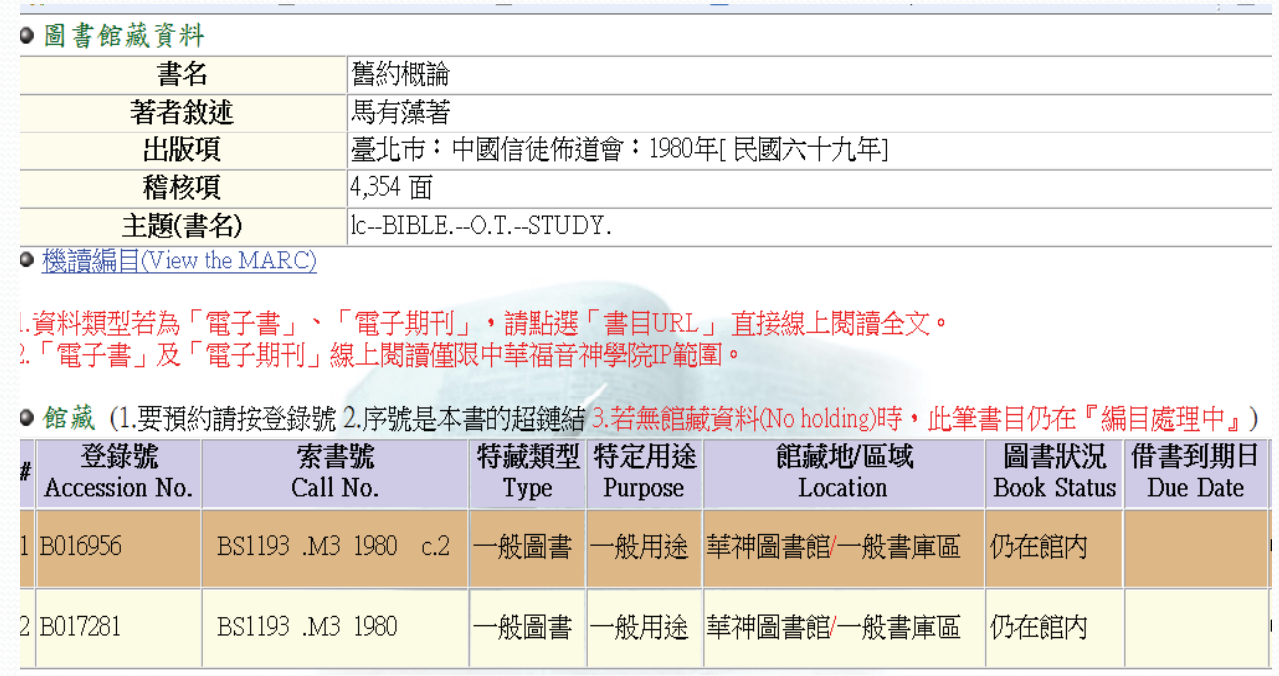

\section{Resources}

General information on CJK Cataloguing - rules etc.

- Library of Congress http://www.loc.gov/catdir/cpso/CJKIntro.html

- OCLC

http://www.oclc.org/connexion/about/features/nonlatin/

- Librariesaustralia

http://www.nla.gov.au/librariesaustralia/expanding-non-latinscript-cataloguing-in-libraries-aust 


\section{Resources (continue)}

CJK catalogue - useful sites

Universities - ANU, Melbourne U, Macquarie U

http://library.anu.edu.au/search/X

http://cat.lib.unimelb.edu.au/search/

http://www.mq.edu.au/on campus/library/

Theological schools - OCLC, EIN, CES

http://www.oclc.org/au/en/worldcat/default.htm

http://www.cgst.edu/en/int8.html

http://wwwlibe.ces.org.tw/webpac2/msearch.dll

\section{Thank you!}

My contact details:

ctcalibrary@gmail.com

Phone: (02)9715 2992

Mobile: 0434353173 